\author{
C. H. Venner \\ University of Twente, \\ Faculty of Mechanical Engineering, \\ Department of Engineering Fluid Dynamics, \\ P.0. Box 217, \\ 7500 AE Enschede, \\ The Netherlands \\ G. Berger \\ Laboratoire de Mécanique des Contacts, \\ UMR CNRS 5514, \\ INSA de Lyon, \\ 20 Ave. A. Einstein, \\ 69621, Villeurbanne Cedex, \\ France \\ P. M. Lugt \\ SKF Engineering \& Research Centre, \\ P.0. Box 2350, \\ 3430 DT Nieuwegein, \\ The Netherlands
}

\section{Waviness Deformation in Starved EHL Circular Contacts}

By means of numerical simulations the deformation of transverse and isotropic harmonic waviness in EHL circular contacts under pure rolling has been studied in relation to the lubricant supply to the contact. In earlier work the deformation of waviness under pure rolling in a fully flooded contact was shown to depend on a single non-dimensional wavelength parameter. In terms of this parameter short wavelengths deform very little. In this paper the effect of starvation on this behavior is shown. First, the steady state smooth surface problem is discussed as an introduction and as a reference problem. It is illustrated in detail how the entire film thickness level decreases with decreasing lubricant supply. Subsequently, results are presented for the time dependent problem with waviness moving through the contact under pure rolling. The relative deformed amplitude of the waviness inside the contact is shown to depend on the same non-dimensional wavelength parameter as before, but also on the degree of starvation. A smaller lubricant supply leads to a larger reduction of the waviness amplitude inside the contact. Finally, it is shown that to an acceptable accuracy the relative deformed amplitude of the starved problem can be predicted by the formula for the fully flooded problem if the generalized wavelength parameter is modified using the reduction factor of the central film thickness for the starved steady state smooth contact. For this reduction factor an accurate formula is available and as a result also for starved contacts by means of a component wise approach a crude estimate of the deformed surface micro-geometry (roughness) inside a contact can be obtained quite easily now. [DOI: 10.1115/1.1572514]

\section{Introduction}

In theoretical studies of EHL contacts it is common to assume that the inlet to the contact is fully flooded: The point where pressure generation starts is assumed to be (or taken) far away from the Hertzian contact. In analytical studies this inlet boundary position is taken infinetely far away. In numerical simulations some finite domain must be taken, which is chosen such that the inlet boundary is sufficiently far from the contact so as to approximate the fully flooded situation for which the generated film thickness reaches its maximum value. However, when looking at the conditions under which most EHL contacts operate in practice, e.g. in bearings, it seems far from realistic to assume that these contacts are fully flooded.

Firstly, most bearings are lubricated with grease. Modelling different aspects of grease lubrication is extremely difficult. Much experimental research is still needed to identify the different (physical) mechanisms that may play a role and the complicated rheological behavior of grease and the changes of its properties in time increases the complexity of the matter. One of the possible mechanisms is that in the initial overrollings most of the grease is pushed to the side and a layer of thickener or worked grease is left on the track. The long-life performance is then determined by a situation in which this pushed aside grease acts as a reservoir which somehow releases base-oil to the track, which is then used to generate a film. Clearly the (base oil) supply to the contact will then depend on the speed conditions (time between overrollings) and it seems unlikely that it will be accurately represented by a fully flooded inlet condition. The second reason why fully flooded conditions may be rare in practice is that, even if the bearing is lubricated with oil, the amount of oil in the bearing is often limited as too much oil would lead to unacceptably large frictional losses, e.g., by churning.

From the above it can be concluded that the input to a given contact often is not a real pool of lubricant which would ensure a

Contributed by the Tribology Division for publication in the ASME JOURNAL OF TRIBOLOGY. Manuscript received by the Tribology Division September 11, 2002 revised manuscript received December 30, 2002. Associate Editor: R. W. Snidle. fully flooded inlet. A more realistic assumption is that the inflow to the contact is the combined result of a layer of oil left behind on the track by the previous contact (possibly on top of a residual layer of thickener or worked grease) and some reflow mechanisms acting in the time between overrollings. These reflow mechanisms depend on the operating conditions and for high speeds, or thin layers, the contacts do not attain the fully flooded film thickness because of "starvation:" The supply of lubricant is such that the starting point of the pressure generation is so much closer to the contact that the generated film thickness is significantly smaller than the value one would expect based on fully flooded theory. The occurrence of "starvation" for high speeds can be clearly illustrated experimentally on a ball on disk apparatus, see $[1,2]$. With increasing speed the film thickness increases until beyond a certain speed the curve levels off and even decreases with increasing speed.

The insights given above are the result of many studies of the starved lubrication problem and grease lubrication. The reader is referred to [3] and [4] for a more detailed overview and references. One of the earliest studies of the starved EHL problem was carried out by Wedeven et al. [5]. In their work the film thickness reduction due to starvation is related to the inlet conditions, in particular the inlet location and inlet film thickness. Other characteristic early works are the work of Chiu [1] and related to starvation is the work of Pemberton and Cameron [6] in which the flow around and into the contact is illuminated. The starved EHL problem was studied theoretically using a Grubin line contact type of analysis by Wolveridge, [7], and numerical simulation results for point contacts were presented by Ranger et al. [8] and Hamrock and Dowson [9]. However, in these works the inlet location itself is taken as the input variable. The advantage is that the problem can then be analyzed with the standard solvers for the fully flooded problem. However, the resulting film reduction formulas are not so well suited to predict the film reduction due to starvation in a practical situation because the inlet is a free boundary and its location will depend on the operating conditions. An algorithm that facilitates the simulation of starvation/cavitation problems with a free boundary has been presented by Elrod $[10,11]$. Such a model was used by Chevalier, Lubrecht and Cann 
in a study of the starved steady state EHL circular contact problem. Their work emphasizes the relevance of the starved problem for understanding grease lubricated contacts, see [12-14,15]. A very practical result is a formula predicting the film thickness reduction due to starvation in relation to the thickness of the lubricant layer supplied to the contact, see [14].

So far all studies of the starved problem were restricted to the steady state situation. The transient problem was first addressed by Wijnant $[16,17]$ who studied the influence of starvation on the dynamics (stiffness and damping) of the EHL contact. In this study the surfaces were assumed to be perfectly smooth. A logical next step is to study the influence of surface features or surface roughness on the performance of starved contacts, e.g., see Dumont et al. [18], where the influence of certain specific features (pits) on the film formation is investigated. A systematic approach to the deformation of waviness under starved conditions is the subject of this paper.

In earlier work [19-21] for the fully flooded problem it was shown that there is a unifying mechanism which controls deformation of waviness in EHL contacts. Under pure rolling the deformation of harmonic waviness is as a function of a single dimensionless parameter representing the ratio of waviness wavelength to inlet length of the contact [22,23]. It can be seen as a generalized wavelength parameter. In terms of this parameter high frequency components deform very little whereas low frequency components are almost completely deformed. The behavior was shown to occur in line and point contacts, and equally for transverse, isotropic, and longitudinal patterns. The results could be represented in a simple formula which can be used as an engineering tool to predict a deformed surface microgeometry given the operating conditions and undeformed microgeometry by applying it to each of the Fourier components of the (measured) microgeometry and subsequently computing the inverse Fourier transform, see Masen et al. [24].

The aim of the present work is to investigate how the deformation of waviness in a circular EHL contact under pure rolling conditions is effected by starvation. For this purpose numerical simulations have been carried out measuring the deformation of transverse and isotropic waviness under pure rolling as a function of load conditions, wavelength, pattern orientation, and degree of starvation.

\section{Equations}

The model used here to describe the starved EHL contact is the model of Chevalier et al., see [12-14,15], but extended to account for transient effects. The complete model is described in detail in [16] and has been succesfully applied to the study of the dynamics of a starved contact, see $[16,17]$. This starvation/cavitation model is based on the work of Elrod [10,11]. An additional variable $\theta$ is introduced representing the degree to which the lubricant film is filled with oil, the so-called fractional film content. If $\theta<1$ the contact is (locally) starved and the pressure equals the cavitation pressure. The variable $\theta$ can be included in the Reynolds equation, see Bayada et al. [25] leading to a single equation that serves as an equation for the pressure in pressurized regions, and as a couette flow mass conservation equation for $\theta$ in the cavitated/starved regions.

In terms of the dimensionless variables defined in the nomenclature the Reynolds equation for the starved time-dependent problem is then given by:

$$
\frac{\partial}{\partial X}\left(\frac{\bar{\rho} H^{3}}{\bar{\eta} \bar{\lambda}} \frac{\partial P}{\partial X}\right)+\frac{\partial}{\partial Y}\left(\frac{\bar{\rho} H^{3}}{\bar{\eta} \bar{\lambda}} \frac{\partial P}{\partial Y}\right)-\frac{\partial(\bar{\rho} \theta H)}{\partial X}-\frac{\partial(\bar{\rho} \theta H)}{\partial T}=0
$$

with

$$
\bar{\lambda}=\frac{6 \eta_{0} u_{s} R_{x}^{2}}{a^{3} p_{h}}
$$
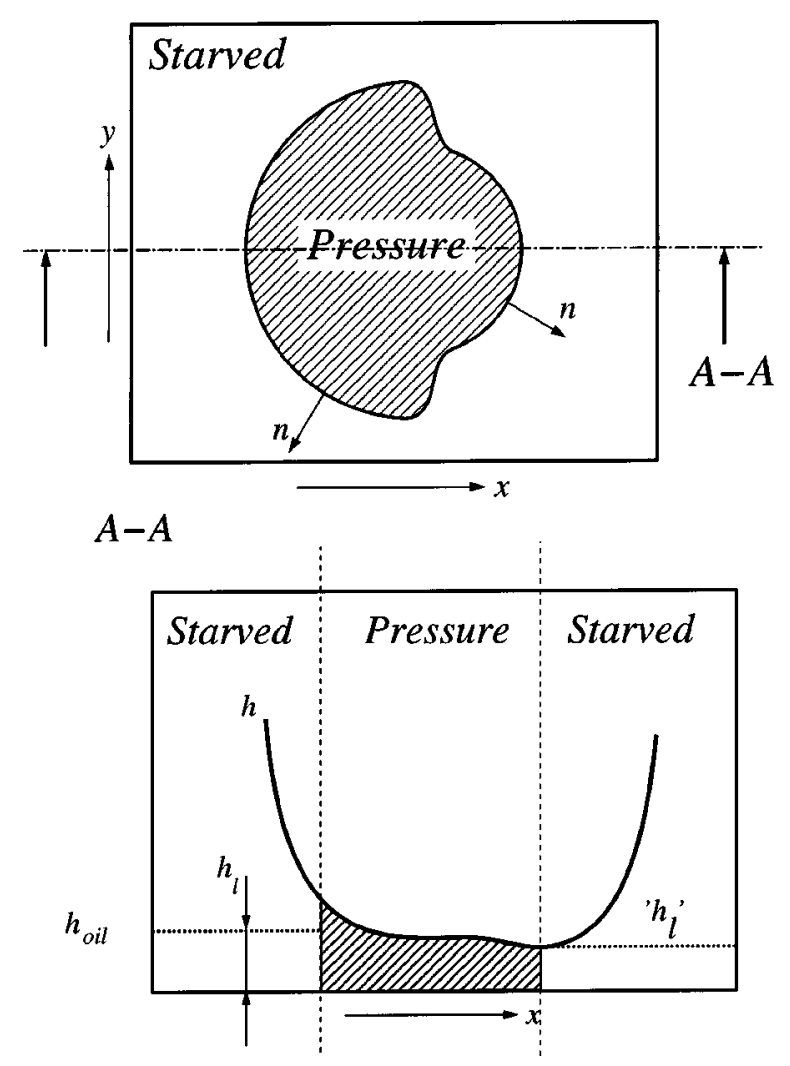

Fig. 1 Illustration of pressurized and cavitated/starved region in an EHL contact with limited lubricant supply at the inlet

The viscosity $\bar{\eta}$ will be taken according to the Roelands equation [26] using $\alpha=1.710^{-8}\left[\mathrm{GPa}^{-1}\right]$ and $\eta_{0}=8.910^{-3}$ [Pa.s]. The density $\bar{\rho}$ is assumed to depend on the pressure according to the Dowson and Higginson equation [27].

To obtain a unique solution, Eq. (1) is subjected to the complementarity condition:

$$
P(X, Y, T)(1-\theta(X, Y, T))=0
$$

with

$$
P(X, Y, T) \geqslant 0
$$

and

$$
0<\theta(X, Y, T) \leqslant 1
$$

With these conditions Eq. (1) is an equation for $\theta$ with $0<\theta$ $<1$ and $P=0$ in the "starved" or "cavitated" regions. In pressurized regions it is an equation for $P>0$ with $\theta=1$. Note that Eq. (1) for $\theta=1$ simplifies to the usual Reynolds equation. In earlier studies starved lubricant supply was simulated by varying the location of the inlet boundary for a fully flooded model, see $[8,9]$. However, in reality the boundary between the starved/cavitated and pressurized region is a free boundary with its location depending on the amount of lubricant supplied and the operating conditions. This is physically more realistic. In Fig. 1 a characteristic shape of the free boundary around an EHL contact is shown. Also shown is a cross-section of the film at the centerline of the contact. It is assumed that in the inlet region a layer of oil with a certain thickness is present. The thickness and the shape of this layer and the load conditions determine the location and shape of the boundary between the starved/cavitated and pressurized region. This behavior can be simulated with the presented model.

At the free boundary between a cavitated/starved and pressurized region a mass flow continuity condition can be derived which should be satisfied (in a numerical algorithm upon convergence 
with decreasing mesh size). This condition is known as the Jakobsson-Floberg-Olsson (JFO) relation, see [28,29]. For a detailed discussion and its derivation for the transient two dimensional problem see $[16,17]$.

The boundary conditions for Eq. (1) are the condition of ambient pressure at the boundary of the domain: $P(X, Y, T)=0$ for $X$ $=X_{a}, X=X_{b}, Y=Y_{a}$ and $Y=Y_{b}$, as for the fully flooded problem, and an additional equation to determine the value of $\theta$ at the inlet boundary $X=X_{a}$. One way is to prescribe $\theta(Y)$ but it is physically more realistic to assume that a layer of oil with a certain thickness and shape is present at the surface in the inlet: $H_{\text {oil }}(Y)$, as schematically drawn in Fig. 1 . In that case the equation for $\theta$ at the boundary is:

$$
\theta\left(X_{a}, Y, T\right)=\frac{H_{\mathrm{oil}}(Y)}{H\left(X_{a}, Y, T\right)},
$$

with $H_{\text {oil }}(Y)$ given. In this equation the value of $H$ at the boundary $X_{a}$, as all values of $H$, is an unknown to be solved from the dimensionless film thickness equation:

$$
\begin{aligned}
H(X, Y, T)= & -\Delta(T)+\frac{X^{2}}{2}+\frac{Y^{2}}{2}+\mathcal{W}(X, Y, T) \\
& +\frac{2}{\pi^{2}} \iint_{S} \frac{P^{\prime}\left(X^{\prime}, Y^{\prime}, T\right) d X^{\prime} d Y^{\prime}}{\sqrt{\left(X-X^{\prime}\right)^{2}+\left(Y-Y^{\prime}\right)^{2}}}
\end{aligned}
$$

where $\Delta$ is the dimensionless mutual approach of the two bodies and $\mathcal{W}$ is a function representing the undeformed roughness of the surfaces. In this study a harmonic waviness on only one surface is assumed. In that case $\mathcal{W}$ can be given as:

$$
\mathcal{W}(X, Y, T)=\mathcal{A} \cos \left(2 \pi \frac{X-\bar{X}}{\left(\lambda_{x} / a\right)}\right) \cos \left(2 \pi \frac{Y}{\left(\lambda_{y} / a\right)}\right)
$$

where $\bar{X}=X_{s}+S T$ with $X_{s}$ the start position of the waviness, $S$ $=u_{1} / \bar{u}=1+\Sigma / 2$ with $u_{1}$ the velocity of the wavy surface, and:

$$
\mathcal{A}=A_{i} 10^{-10\left(\max \left(0, X-\bar{X} /\left(\lambda_{x} / a\right)\right)^{2}\right)}
$$

This particular shape was chosen to avoid discontinuous derivatives, and to start the calculation with a smooth surface geometry. $A_{i}$ denotes the amplitude of the undeformed waviness. The deformed amplitude of the pattern as a function of the operating conditions will be determined from the film thickness oscillations according to:

$$
2 A_{d}=\max _{T} H(0,0, T)-\min _{T} H(0,0, T)
$$

Finally, an equation of motion is needed which determines the unknown integration function $\Delta(T)$. If acceleration terms are neglected this equation reduces to the usual condition of force balance:

$$
\frac{3}{2 \pi} \iint_{S} P(X, Y, T) d X d Y=1 \quad \forall T
$$

\section{Numerical Solution}

The Eqs. (1)-(10) were discretized using a second order discretization in space and time. The resulting discrete equations at each timestep were solved iteratively using Multigrid techniques where the relaxation process around which the solver is built is a mixed distributive Jacobi and Gauss-Seidel line relaxation scheme. The complementarity condition is incorporated in the relaxation scheme. For the fast evaluation of the discrete elastic deformation integrals Multilevel Multi-Integration [30] was used. For a detailed description of the basic elements of a Multigrid solution algorithm for the EHL circular contact problem the reader is referred to [31].

Most calculations where carried out using a domain $-2.5 \leqslant X$ $\leqslant 1.5$ and $-2 \leqslant Y \leqslant 2$. For low loads and a lubricant supply close to fully flooded a larger domain was used: $-4.5 \leqslant X \leqslant 1.5$ and $-3 \leqslant Y \leqslant 3$. The steady state results presented in section 5 were obtained using grid with $1025 \times 1025$ points.

For the time dependent results presented in Sec. 6 two different second order discretization were used: For larger wavelengths a standard second order upstream discretization of the wedge and squeeze term denoted by SU2. For small wavelengths a narrow upstream second order discretization (NU2). In this scheme the wedge and squeeze terms are treated in a combined way as a result of which it has a zero second order term in the truncation error for several directions including the characteristic direction $X=T$. Consequently, on a given grid the artificial diffusivity is smaller and the result will be more accurate as will be illustrated. This is particularly important when the amplitude of film thickness oscillations inside the contact region are measured and the wavelength of the waviness is small. The calculations for the transient problem were carried out using a grid with $257 \times 257$ points and a timestep equal to the mesh size. This latter choice is optimal from the viewpoint of minimizing the error for the characteristic direction $X=T$ for both NU2 and SU2 schemes. For more details regarding the two schemes the reader is referred to $[16,32]$.

\section{Steady State: Smooth Surfaces}

First the effect of an increasing degree of starvation on the film thickness and pressure in the steady state case with ideally smooth surfaces is illustrated. These steady state results will serve as a reference from which the results for the transient problem to be presented in the next section can be understood. The load condition is characterized by the values of the Moes dimensionless parameters $M=20$ and $L=10$. If the surfaces are made of steel and the lubricant is a standard mineral oil this represents a moderately loaded contact with a maximum Hertzian pressure of about $0.5 \mathrm{GPa}$. This case was chosen because for a moderately loaded contact the changes in the solutions with increasing degree of starvation are more easy to see in the graphs. The trends to be observed for higher loads are not essentially different, but the changes take place in smaller regions, see also [3].

Figure 2 shows the computed dimensionless pressure profile and film thickness as a function of $X$ and $Y$ for the case of an inlet oil layer with a thickness that is half the central film thickness for the fully flooded case: $H_{\mathrm{oil}}=H_{c f} / 2$. As for the fully flooded contact the pressure in the central region is roughly the Hertzian dry contact pressure. However, for the fully flooded case the pressure in the inlet gradually rises, whereas for the starved problem in a large part of the inlet region the pressure is zero and the pressure build up suddenly starts at a free boundary. This is due to the fact that pressure generation can only occur at a location once the gap has become sufficiently small for the amount of lubricant to fill it. In other words, a nonzero pressure can only occur when the fractional film content $\theta$ satisfies $\theta \geqslant 1$. This can be seen from comparing the graph of $\theta(X, Y)$ with the graph of $P(X, Y)$. Noted that $\theta$ exhibits a discontinuity at the free boundary on the inlet side. At this point also the pressure gradient is discontinuous. The jump in $\theta$ and the pressure gradient at the free boundary are related. They should satisfy the so-called JFO condition. In the discrete scheme this will be the case upon convergence, i.e., with decreasing mesh size, see $[16,17]$.

The location of the free boundary depends on the degree of starvation. This is shown in detail in Fig. 3 and 4. These figures show cross sections of the solutions obtained with decreasing $H_{\text {oil }}$. The figures show dimensionless pressure $P$, film thickness $H$, and fractional film content $\theta$ as a function of $X$ at $Y=0$ and as a function of $Y$ at $X=0$. With respect to the film thickness the figures show that with decreasing $H_{\text {oil }}$ the entire level of the film thickness, and thus the central film thickness decreases. However, also the film shape changes: the horseshoe shape with side-lobes disappears leading to a nearly uniform film thickness for severely starved contacts. With respect to the pressure the figures show that the smaller the layer of lubricant supplied to the contact, the closer 

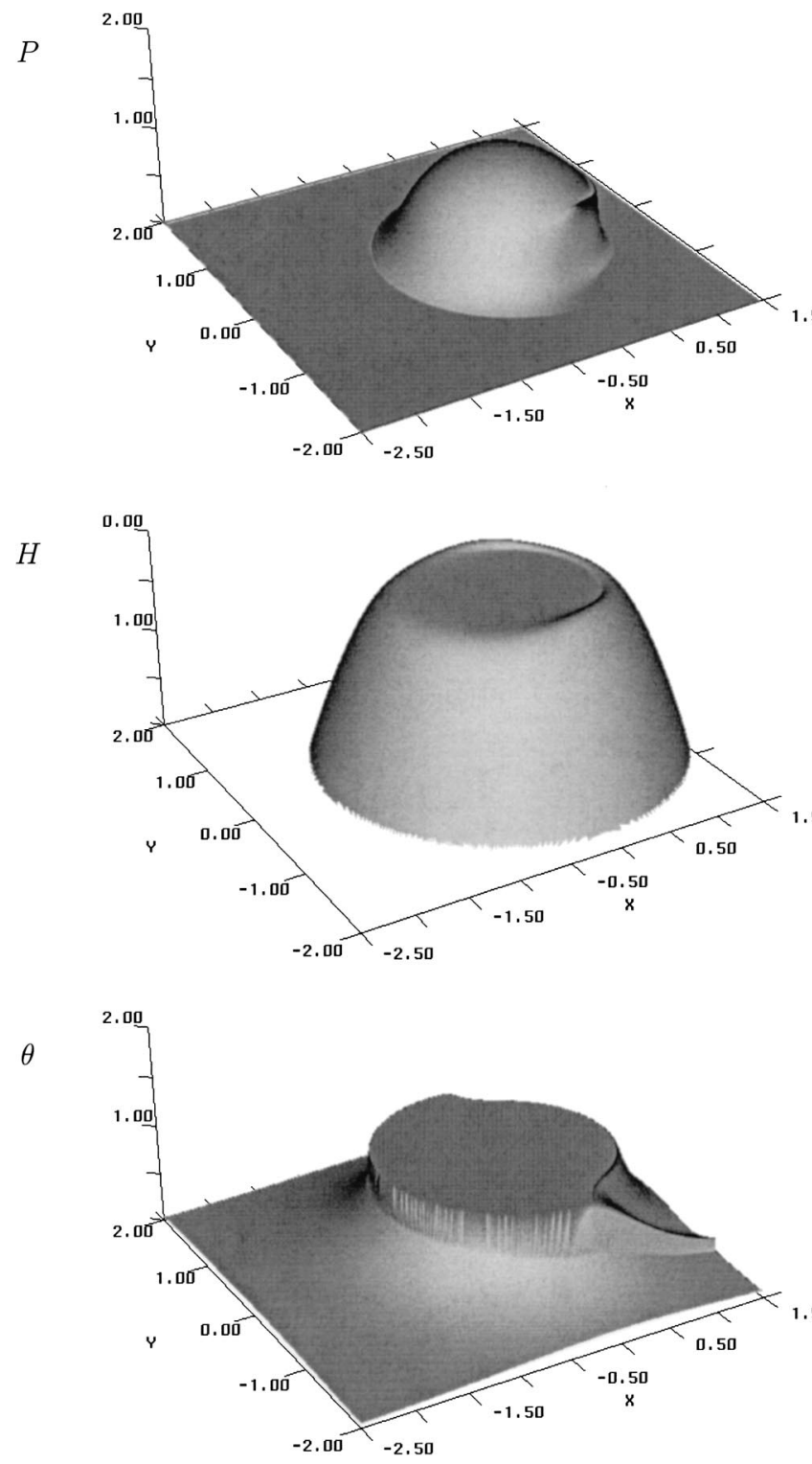

Fig. 2 Dimensionless pressure $\boldsymbol{P}$ (top), film thickness $\boldsymbol{H}$ (center) and fractional film content $\theta$ as a function of $X$ and $Y$ for $M=20, L=10$. Starved contact: $H_{\text {oil }} / H_{c f}=0.5$.

to the edge of the Hertzian contact circle the pressure generation starts, and the larger the pressure gradient at the free boundary. Together with the changes in the pressure spike that can be seen clearly in the figure this illustrates how with decreasing $H_{\text {oil }}$ the pressure profile approaches the Hertzian dry contact pressure. Finally, by the JFO condition the larger gradient and smaller film thickness imply that the jump in $\theta$ at the free boundary will be smaller as can seen from the graphs $\theta(X)$.

Some quantitative results illustrating the numerical accuracy of the steady-state results are given in the Tables 1 and 2. These tables show the value of the computed minimum film thickness as a function of the inlet layer thickness (degree of starvation) and the mesh size of the computational domain. The tables show that the computed values of minimum and central film thickness converge in a second order manner to a limiting value as is consistent with the fact that a second order discretization is used. Also the tables clearly show that the minimum and central film thickness with increasing degree of starvation converge to the same value reflecting the flattening of the film.

The decrease of the central film thickness with decreasing layer
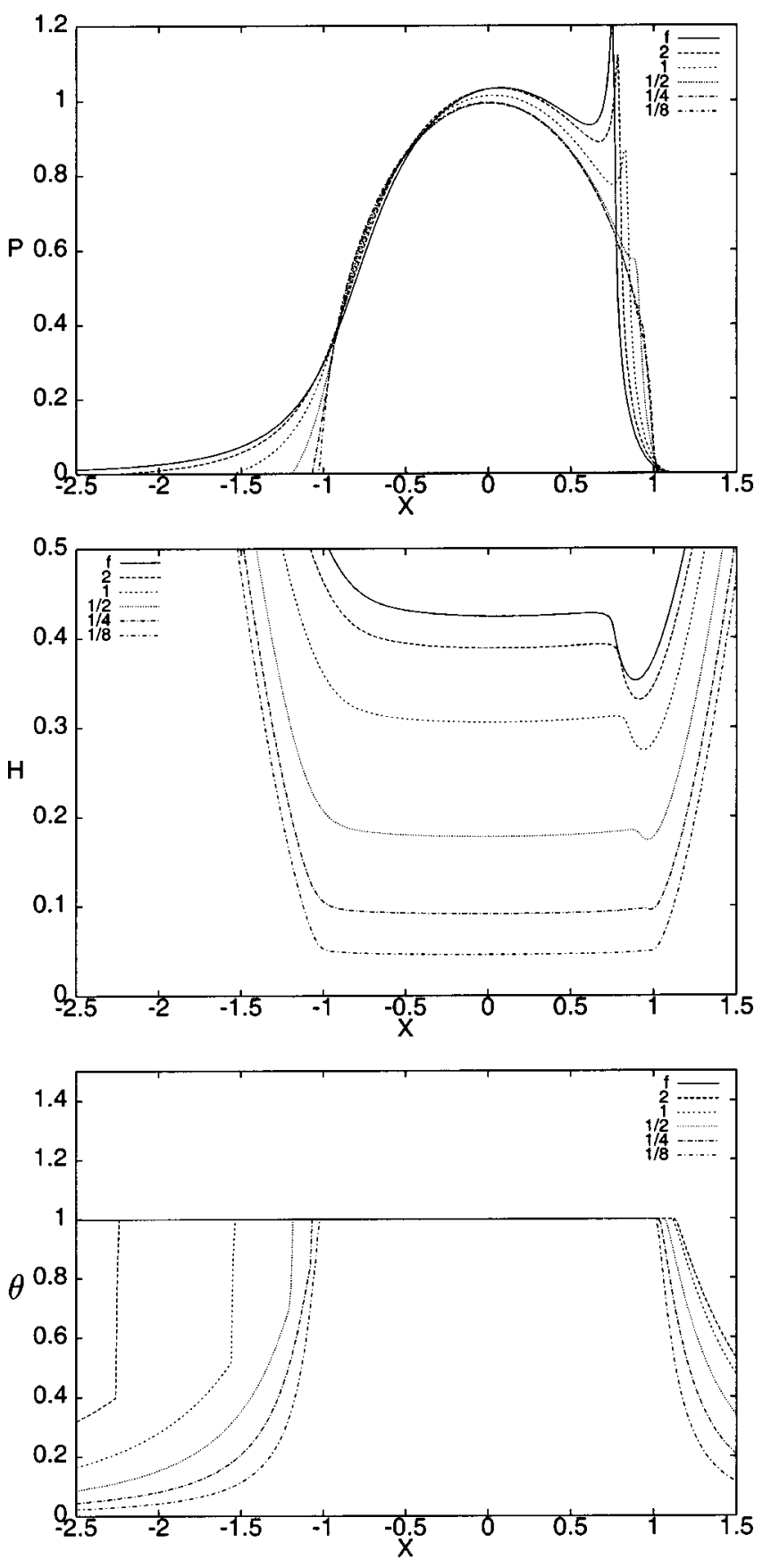

Fig. 3 Dimensionless pressure $P$ (top) and film thickness $H$ (bottom) as a function of $X$ at the centerline $Y=0$ for the case $M=20, L=10$ and $H_{\text {oil }} / H_{c f}=\infty, 2,1,1 / 2,1 / 4$, and $1 / 8$.

thickness has been studied in detail by Chevalier et al. $[3,14]$. They found that the ratio of the central film thickness in the starved contact to the central film thickness under fully flooded conditions could be approximated by the following relation:

$$
\mathcal{R}=\frac{H_{c}}{H_{c f}}=\frac{r}{\sqrt[\gamma]{1+r^{\gamma}}}
$$

where $r=H_{\mathrm{oil}} /\left(\bar{\rho}\left(p_{h}\right) H_{c f}\right)$ and $\gamma$ is a parameter $2 \leqslant \gamma \leqslant 5$. In [33] the behavior of this parameter in relation to the load conditions is studied in detail by looking at starved elliptic contacts.

When plotting the predictions of Eq. (11) two asymptotes can be observed: $r \rightarrow 0$ and $r \rightarrow \infty$ which give $\mathcal{R} \rightarrow r$ and $\mathcal{R} \rightarrow 1$ respectively. Of these two, the asymptote for thin films is the most 

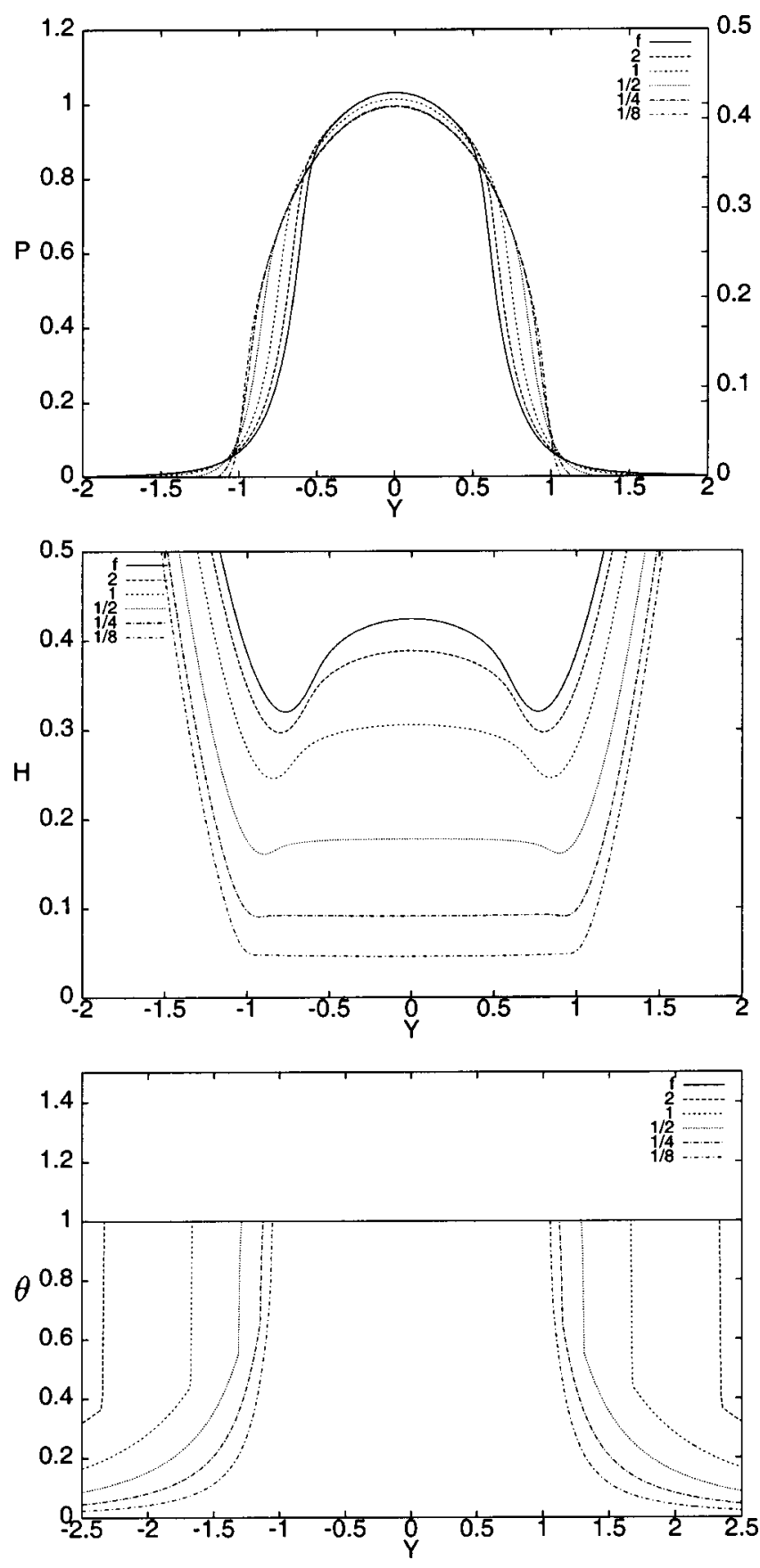

Fig. 4 Dimensionless pressure $P$ (top) and film thickness $H$ (bottom) at the line $X=0$ as a function of $Y$ for the case $M$ $=20, L=10$ and $H_{\text {oil }} / H_{c f}=\infty, 2,1,1 / 2,1 / 4$, and $1 / 8$. interesting, as it shows that for very thin oil films, the EHL film thickness is the same as the film in front of the contact, with a minor correction related to the compressibility. In other words: the contact becomes very efficient in building up an oil film. Almost all available oil is used to generate the film separating the surfaces and the side-leakage is small, whereas in a fully flooded contact most of the oil flows around the contact and only a small portion actually passes through the central region.

The advantage of Eq. (11) is that it can easily be used in practice. One only needs an estimate of $H_{c f}$ but this can be obtained from one of the film thickness formulas as the ones presented by Hamrock and Dowson [34] and Nijenbanning et al. [35].

In the literature alternative prediction formulas for the film thickness reduction in starved contacts have been derived using a Grubin type of analysis, see [5,7]. This approach gives formula relating $H_{c} / H_{c f}$ to the film thickness at the inlet boundary, in this case the free boundary, for which generally no accurate approximation is available. For example, the formulation of the Grubin analysis by Hooke [36] can be modified easily to account for a starved inlet. For that case one obtains:

$$
\frac{H_{c}}{H_{c f}}=\left(\frac{\mathcal{I}\left(H_{b} / H_{c}\right)}{\mathcal{I}(0)}\right)^{3 / 4}
$$

where $H_{b}$ denotes the value of the film thickness at the location of the inlet boundary and $\mathcal{I}$ is defined by:

$$
\mathcal{I}(\omega)=\int_{\omega}^{1} \zeta^{1 / 3}(1-\zeta)^{2 / 3} d y
$$

$\omega=0$ represents a fully flooded contact. The integration can be carried out numerically and it follows that:

$$
\frac{H_{c}}{H_{c f}} \approx 1-\frac{H_{c}}{H_{b}}
$$

When taking the solutions of the starved problem it can be shown that Eq. (14) is quite accurate. However, it is not very well suited for predictions as one needs to know the film thickness at the inlet location, i.e., the free boundary which in general is unknown. Only for the severely starved case this is easy to guess, $H_{b}$ $\approx H_{\text {oil }}$ so if $H_{\text {oil }}=\omega H_{c f}$ one then obtains:

$$
\frac{H_{c}}{H_{c f}}=\frac{\omega}{\omega+1}
$$

As can be verified using the results presented in Table 1, Eq. (11) gives a good prediction of $H_{c} / H_{c f}$. Eq. (15) on the other hand is only accurate $H_{\text {oil }}<H_{c f}$. For larger values of $H_{\text {oil }}$ it underestimates the starved film thickness. This is not because the Grubin analysis is not valid but because for these cases the assumption $H_{b} \approx H_{\text {oil }}$ used to obtain Eq. (15) from Eq. (14) is no longer justified as $H_{b}>H_{\text {oil }}$.

\section{Time Dependent: Waviness}

Next the transient problem with a waviness moving through the contact is addressed. The results presented are restricted to the

Table 1 Convergence of dimensionless central film thickness with decreasing mesh size for different degrees of starvation. $M$ $=20, L=10$

\begin{tabular}{cccccccc}
\hline \hline & \multicolumn{7}{c}{$H_{\text {oil }} / H_{c f}$} \\
\cline { 2 - 7 }$n_{X} \times n_{Y}$ & $\infty$ & 5 & 2 & 1 & $1 / 2$ & $1 / 4$ \\
\hline$(32+1)^{2}$ & $3.75010^{-1}$ & $3.69910^{-1}$ & $3.43510^{-1}$ & $2.85810^{-2}$ & $1.75210^{-1}$ & $8.54610^{-2}$ & $4.00610^{-2}$ \\
$(64+1)^{2}$ & $4.11810^{-1}$ & $4.07010^{-1}$ & $3.76310^{-1}$ & $2.99610^{-1}$ & $1.75310^{-1}$ & $8.98610^{-2}$ & $4.53510^{-2}$ \\
$(128+1)^{2}$ & $4.21210^{-1}$ & $4.16310^{-1}$ & $3.85010^{-1}$ & $3.02310^{-1}$ & $1.75610^{-1}$ & $9.00810^{-2}$ & $4.53510^{-2}$ \\
$(256+1)^{2}$ & $4.23610^{-1}$ & $4.18610^{-1}$ & $3.87110^{-1}$ & $3.03310^{-1}$ & $1.75610^{-1}$ & $9.01410^{-2}$ & $4.53210^{-2}$ \\
$(512+1)^{2}$ & $4.24210^{-1}$ & $4.19210^{-1}$ & $3.87710^{-1}$ & $3.03610^{-1}$ & $1.75610^{-1}$ & $9.01610^{-2}$ & $4.53010^{-2}$ \\
$(1024+1)^{2}$ & $4.24310^{-1}$ & $4.19410^{-1}$ & $3.87810^{-1}$ & $3.03710^{-1}$ & $1.75610^{-1}$ & $9.01510^{-2}$ & $4.53010^{-2}$ \\
\hline \hline
\end{tabular}


Table 2 Convergence of dimensionless minimum film thickness with decreasing mesh size for different degrees of starvation. $M=20, L=10$.

\begin{tabular}{|c|c|c|c|c|c|c|c|}
\hline \multirow[b]{2}{*}{$n_{X} \times n_{Y}$} & \multicolumn{7}{|c|}{$H_{\mathrm{oil}} / H_{c f}$} \\
\hline & $\infty$ & 5 & 2 & 1 & $1 / 2$ & $1 / 4$ & $1 / 8$ \\
\hline$(32+1)^{2}$ & $2.59110^{-1}$ & $2.58010^{-1}$ & $2.37310^{-1}$ & $2.01310^{-2}$ & $1.37610^{-1}$ & $7.60310^{-2}$ & $3.22710^{-2}$ \\
\hline$(64+1)^{2}$ & $2.84310^{-1}$ & $2.81610^{-1}$ & $2.62410^{-1}$ & $2.17910^{-1}$ & $1.42210^{-1}$ & $8.17210^{-2}$ & $4.35310^{-2}$ \\
\hline$(128+1)^{2}$ & $2.890010^{-1}$ & $2.85910^{-1}$ & $2.67910^{-1}$ & $2.21110^{-1}$ & $1.44710^{-1}$ & $8.34010^{-2}$ & $4.52410^{-2}$ \\
\hline$(256+1)^{2}$ & $2.90310^{-1}$ & $2.87310^{-1}$ & $2.68610^{-1}$ & $2.21510^{-1}$ & $1.45210^{-1}$ & $8.37410^{-2}$ & $4.52910^{-2}$ \\
\hline$(512+1)^{2}$ & $2.90510^{-1}$ & $2.87610^{-1}$ & $2.68910^{-1}$ & $2.21710^{-1}$ & $1.45310^{-1}$ & $8.37710^{-2}$ & $4.53010^{-2}$ \\
\hline$(1024+1)^{2}$ & $2.90510^{-1}$ & $2.87610^{-1}$ & $2.68910^{-1}$ & $2.21710^{-1}$ & $1.45310^{-1}$ & $8.37910^{-2}$ & $4.53010^{-2}$ \\
\hline
\end{tabular}

case of pure rolling $\Sigma=0(S=1)$. Figure 5 shows snapshots of the dimensionless pressure profile, film thickness, and fractional film content obtained at a time a transverse waviness is fully inside the contact. The conditions are the same as for the steady state solution presented in Fig. 2. The waviness amplitude was

$P$

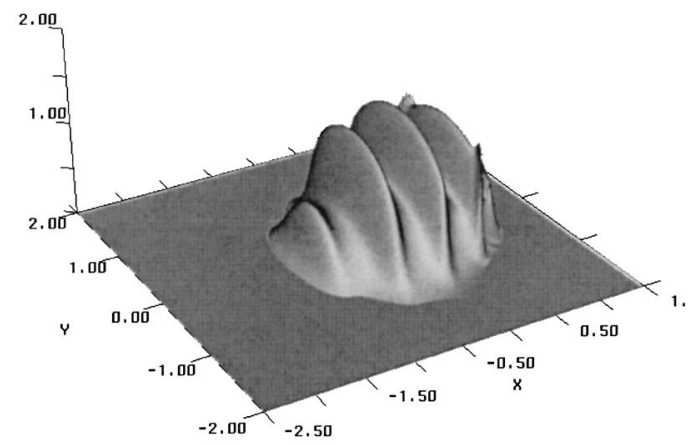

$H$

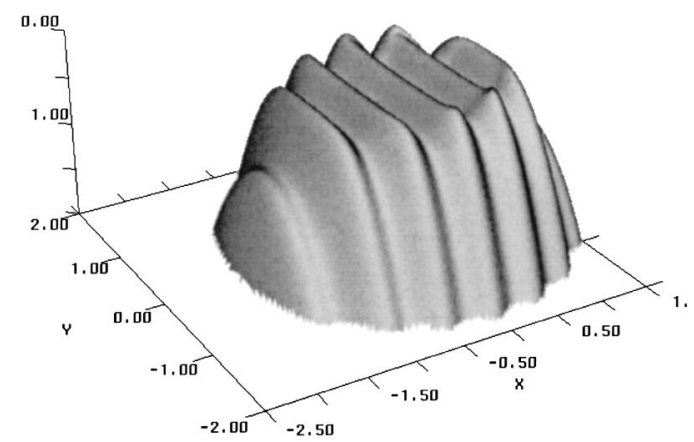

$\theta$

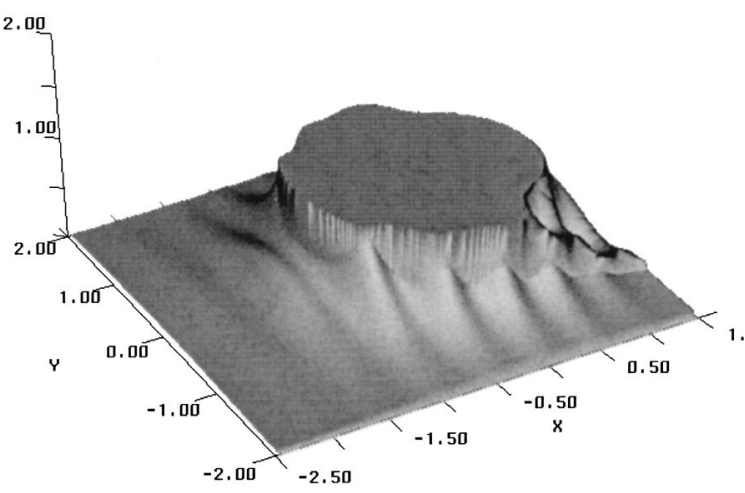

Fig. 5 Snapshot of the dimensionless pressure $P$ (top), and film thickness $H$ (bottom) as a function of $X$ and $Y$ at a given time for $M=20, L=10, \Sigma=0$. Transverse waviness, $\lambda_{x} / a=1 / 2$, $\lambda_{y} / a=\infty$. Starved contact: $H_{\text {oil }} / H_{c f}=0.5$. taken $A_{i}=H_{c f} / 5$ and its dimensionless wavelength $\lambda / a=1 / 2$. In the figure it can be seen that the waviness causes an oscillation of film thickness and pressure in the same way as in the fully flooded problem. The wavelength of the oscillation equals the wavelength of the waviness because of the pure rolling condition. So, the mechanism that the waviness moves through the contact only undergoing a reduction in amplitude is not changed by the introduction of a starved inlet. However, the magnitude of the reduction is effected by the degree of starvation as will be shown in this section.

First an indication of the accuracy of the time dependent results is given. In Table 3 the computed value of $A_{d} / A_{i}$ is given for transverse waviness with a wavelength of $\lambda / a=0.5$ and three values of the starvation parameter $H_{\text {oil }} / H_{c f}$. In the table the values obtained with the two different discretizations SU2 and NU2 of the wedge and squeeze term are shown as a function of the mesh size of the grid (and the timestep). Firstly the table shows that with decreasing mesh size and timestep the value of $A_{d} / A_{i}$ converges. However, for this relatively short wavelength it can be seen clearly that convergence only sets in once the grid is sufficiently fine. Note that the results obtained with the $S U 2$ scheme tend to approach the limiting value from below. This is explained by the artificial diffusivity of the scheme as a result of which on coarse grids it exhibits amplitude decay of the waviness inside the contact, and when taking the value of $A_{d}$ from the variations of $H(0,0)$ it is easily underestimated. The results obtained with the $N U 2$ scheme tend to approach the limiting value from above. This implies that for cases where the waviness deformation is small it tends to be much more accurate. This particularly applies to cases close to fully flooded and for small wavelengths. However, the results presented in the table also show that for the starved problem the NU2 results are not always more accurate. If the waviness deformation is large, as is the case for a small value of $H_{\text {oil }}$ the $N U 2$ scheme tends to underestimate the deformation, or to overestimate the value of $A_{d}$. This can be seen in the results obtained for $H_{\mathrm{oil}}=H_{c f} / 4$. Finally, the table shows that the tendency of the waviness deformation to increase with decreasing $H_{\text {oil }}$ is a real trend as the changes of $A_{d} / A_{i}$ going from $H_{\mathrm{oil}}=H_{c f}$ to $H_{\mathrm{oil}}$ $=H_{c f} / 2$ and from $H_{\mathrm{oil}}=H_{c f} / 2$ to $H_{\mathrm{oil}}=H_{c f} / 4$ are significantly larger than the error in the value of $A_{d} / A_{i}$ that can be estimated from comparing the results obtained on the finest grid and the next coarser grid.

The trend of increasing waviness deformation with increasing degree of starvation (decreasing $H_{\mathrm{oil}}$ ) is illustrated graphically in Fig. 6. The figure shows the computed dimensionless pressure as a function of $X$ at the line $Y=0$ for different values of $H_{\text {oil }} / H_{c f}$. The figure shows that with decreasing layer thickness the level of the film thickness decreases as was found for the steady state case. For that problem it was found that the film thickness became more uniform with increasing degree of starvation. In the transient problem the same behavior is found which is now reflected in a decreasing amplitude of the film oscillations with decreasing value of $H_{\text {oil }} / H_{c f}$. Figure 6 illustrates that also the time dependent solution for waviness with increasing degree of starvation approaches the dry contact solution where the waviness would be 
Table 3 Convergence of relative amplitude of film thickness oscillations $A_{d} / A_{i}$ as a function of mesh size (number of gridpoints). Timestep $h_{T}=h_{X}=h_{Y}$. Conditions $M=20, L=10, \Sigma=0, H_{\text {oil }} / H_{c f}=1,1 / 2$ and 1/4. Transverse waviness $\lambda_{x} / a=1 / 2, \lambda_{y} / a=\infty$.

\begin{tabular}{|c|c|c|c|c|c|c|}
\hline \multirow[b]{3}{*}{$n_{X} \times n_{Y}$} & \multicolumn{6}{|c|}{$H_{\text {oil }} / H_{c f}$} \\
\hline & \multicolumn{2}{|c|}{1} & \multicolumn{2}{|c|}{$1 / 2$} & \multicolumn{2}{|c|}{$1 / 4$} \\
\hline & $S U 2$ & NU2 & $S U 2$ & $N U 2$ & SU2 & $N U 2$ \\
\hline$(32+1)^{2}$ & 0.31 & 0.99 & 0.26 & 0.98 & 0.28 & 0.99 \\
\hline$(64+1)^{2}$ & 0.46 & 0.97 & 0.36 & 0.77 & 0.18 & 0.69 \\
\hline$(128+1)^{2}$ & 0.79 & 0.98 & 0.58 & 0.78 & 0.30 & 0.38 \\
\hline$(256+1)^{2}$ & 0.95 & 0.99 & 0.74 & 0.79 & 0.32 & 0.32 \\
\hline
\end{tabular}

deformed completely. Note that associated with the increasing deformation of the waviness is an increase of the amplitude of the pressure oscillations. Finally, in all cases shown the initial amplitude $A_{i}$ was taken the same: $A_{i}=0.2 H_{c f}$. For the small values of $H_{\mathrm{oil}} / H_{c f}$ this implies that $A_{i}$ is no longer small compared to $H_{c}$ as a result of which the waviness is not only subjected to a reduction in amplitude but due to nonlinear effects also changes shape.

For the fully flooded problem it was shown that the ratio $A_{d} / A_{i}$ depends only on a generalized wavelength defined as:

$$
\nabla=c(\lambda / a)(\bar{\alpha})^{3 / 2} L^{-2}
$$

with $c=\sqrt{\left(2 \pi^{3}\right) / 3}$ for elliptic contacts and $c=(2 \pi)^{3 / 4}$ for line contact. The parameter $\nabla$ can be interpreted as the ratio of the waviness wavelength to the "entrainment length" of the contact, see $[22,23]$. To a good approximation the value of $A_{d} / A_{i}$ for a given case can be obtained from:
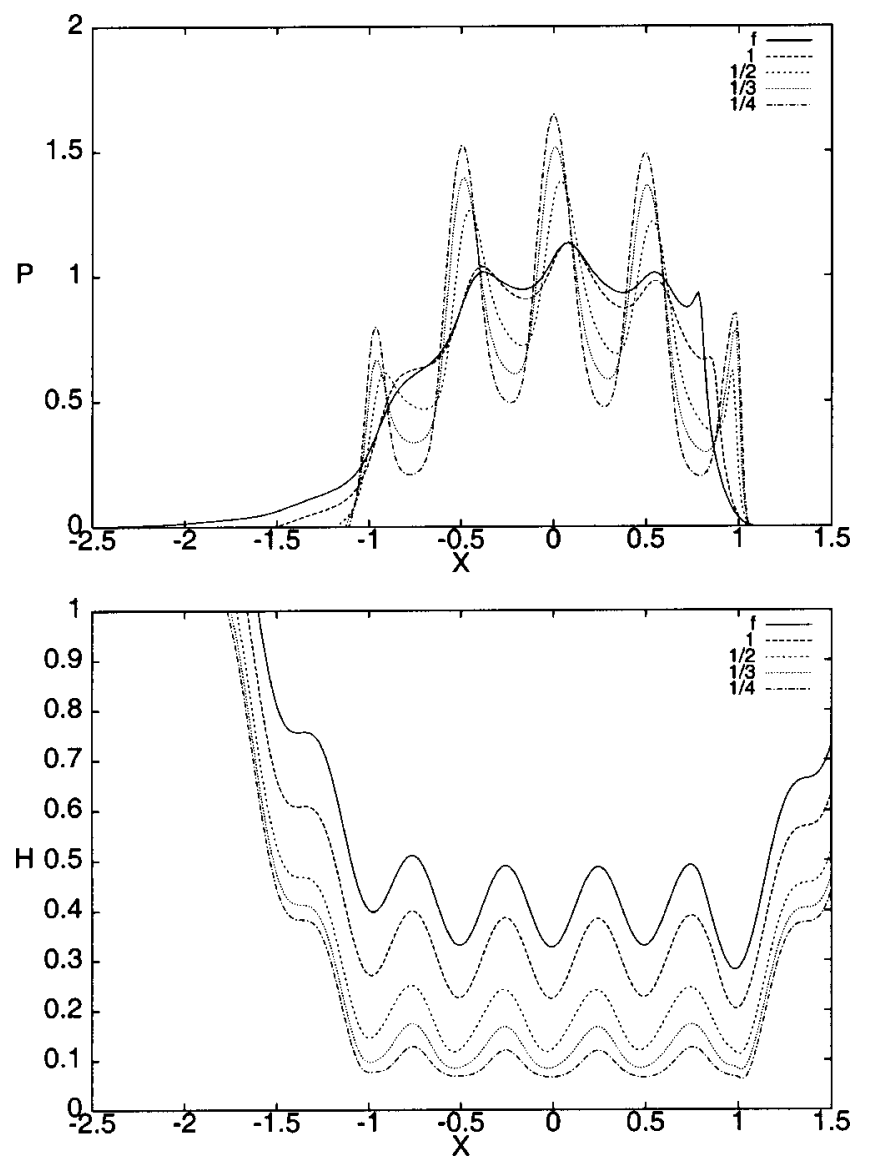

Fig. 6 Dimensionless pressure (top) and film thickness (bottom) as a function of $X$ at the centerline of the contact $Y=0$ for different $H_{\text {oil }} . M=20, L=10, \Sigma=0$. Transverse waviness, $\lambda_{x} / a$ $=1 / 2, \lambda_{y} / a=\infty$. Starved contact: $H_{\text {oil }} / H_{c f}=\infty, 1,1 / 2,1 / 3,1 / 4$.

$$
\frac{A_{d}}{A_{i}}=\frac{1}{1+0.15\left(f\left(\lambda_{y} / \lambda_{x}\right) \nabla\right)+0.015\left(f\left(\lambda_{y} / \lambda_{x}\right) \nabla\right)^{2}}
$$

where $f\left(\lambda_{y} / \lambda_{x}\right)$ is a correction factor for the anisotropy of the waviness. For $\lambda_{y} \geqslant \lambda_{x}: f \equiv 1$ and $\lambda=\lambda_{x}$.

Below it is shown how this behavior is effected by starvation. Simulations have been performed for a range of values of $M, L$, the wavelength $\lambda / a$, and different values of the starvation parameter $H_{\text {oil }} / H_{c f}$. Figure 7 shows the computed values of $A_{d} / A_{i}$ presented a function of $\nabla$ and $H_{\text {oil }} / H_{c f}$ for transverse and isotropic waviness. For the same loading cases results are presented for the fully flooded case, and values $H_{\mathrm{oil}} / H_{c f}=5,2,1,1 / 2$ and $1 / 4$. The drawn line in the figure gives the predictions according to equation (17). The figure shows that for $H_{\mathrm{oil}} \geqslant 2$ the values are close to the values for the fully flooded problem. Subsequently, with decreasing $H_{\mathrm{oil}} / H_{c f}$, a clear tendency of decreasing $A_{d} / A_{i}$
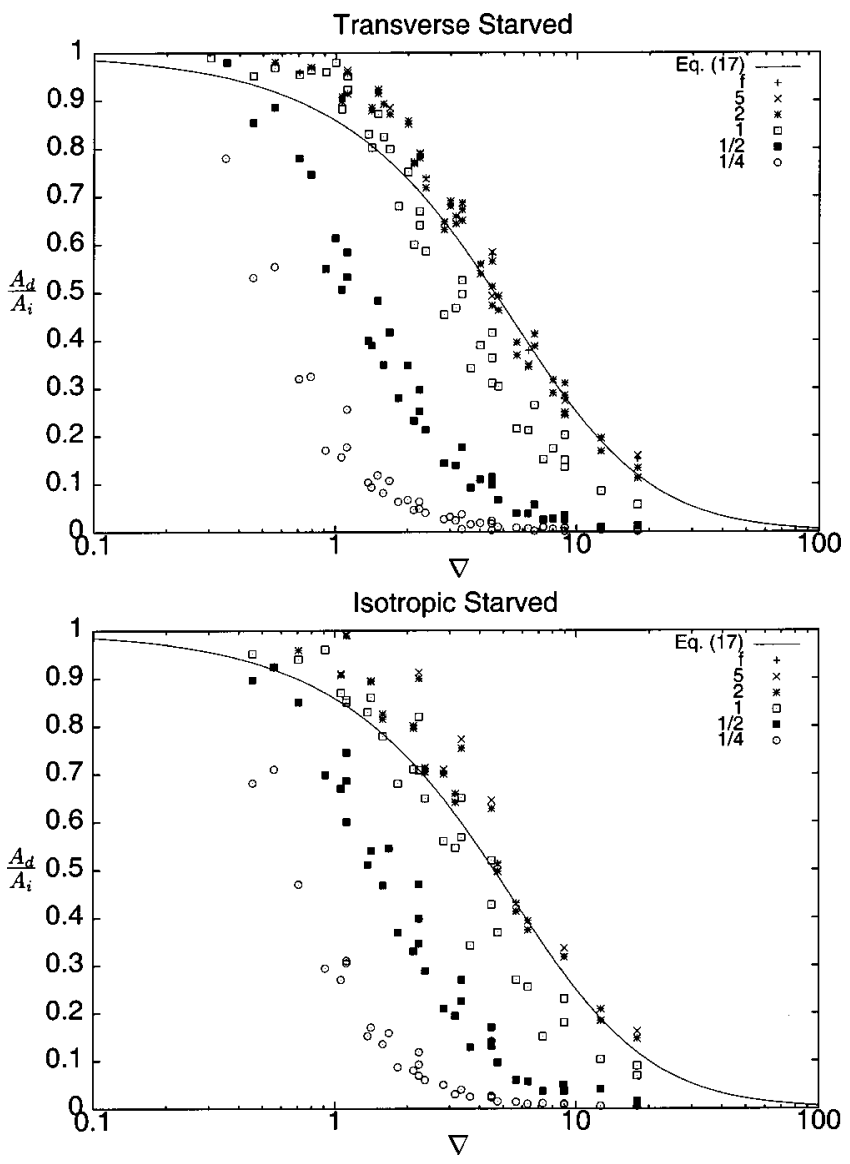

Fig. 7 Relative deformed amplitude $\boldsymbol{A}_{d} / \boldsymbol{A}_{i}$ as a function of $\nabla$ $=c(\lambda / a)(\bar{\alpha})^{3 / 2} / L^{-2}$ and $H_{\text {oil }} / H_{c f}$ for transverse (top) and isotropic (bottom) waviness in a starved contact. The drawn line represents the predictions of Eq. (17). 

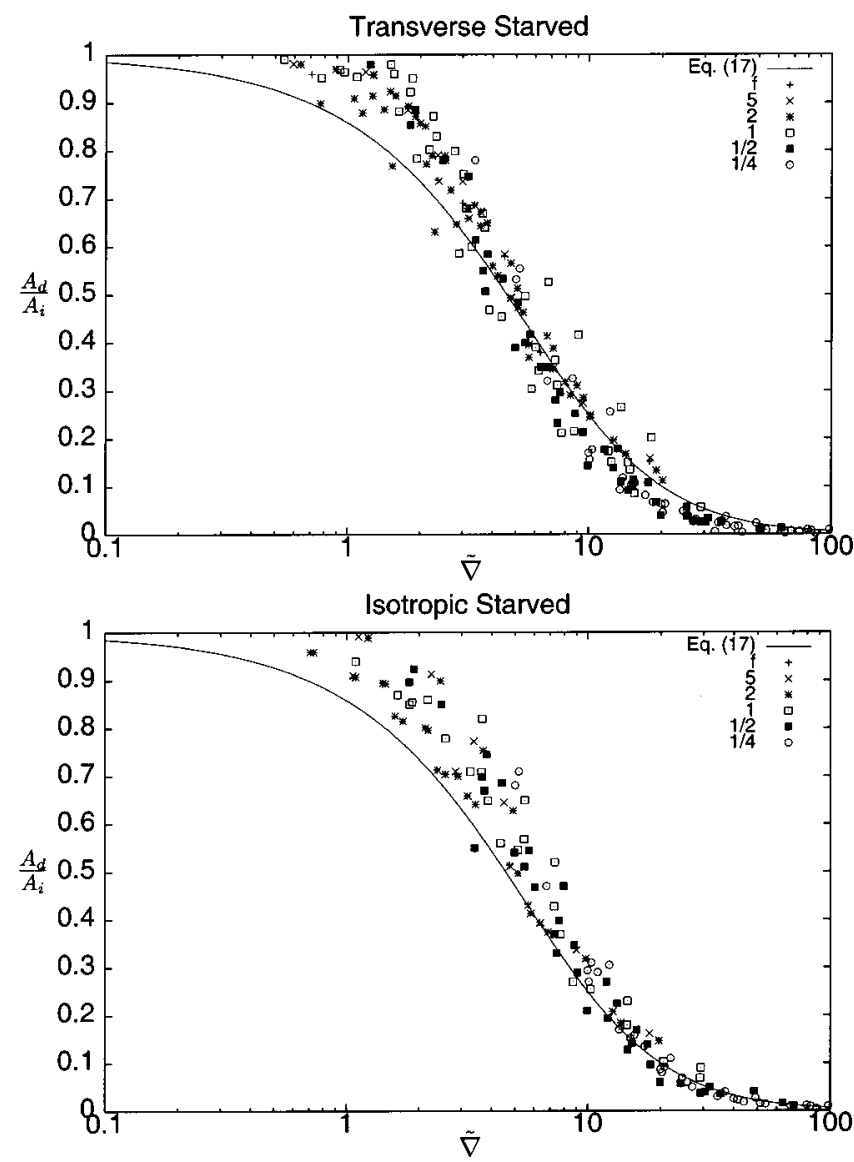

Fig. 8 Relative deformed amplitude $\boldsymbol{A}_{d} / \boldsymbol{A}_{i}$ as a function of $\tilde{\nabla}$ $=\left(H_{c f} / H_{c}\right)^{3 / 2} \nabla$ for transverse (top) and isotropic (bottom) waviness. The drawn line represents the predictions of Eq. (17) with $\nabla$ replaced by ñabla.

can be seen, or, as was shown for a single case in Fig. 3, the more starved the contact, the larger the waviness deformation. Note that for each value of $H_{\text {oil }} / H_{c f}$ a curve similar to the fully flooded curve is obtained but shifted by a certain amount. The same applies to the results for isotropic waviness.

The logical first explanation for the observed behavior seems to be that starvation reduces the inlet length of the contact. As a result the ratio wavelength to entrainment length increases. So, the "effective" value of $\nabla$ for a starved contact is larger than for a fully flooded contact under the same conditions and the deformation of the waviness will be larger. However, this is only part of the explanation. When a $\nabla$ is used obtained correcting for the shorter entrainment length of the starved contact, which can be computed from a Grubin analysis of the type given in Section 5, the results do shift in the right direction but the correction is too small. The correction then given in fact assumes that the starved contact is characterized by a fixed inlet film thickness. However, it should be noted that in the dynamic contact neither the film thickness at the inlet location nor the inlet position are fixed because the inlet moves. When a wavy pattern enters the contact the inlet location shifts towards the center when when a valley enters and it shifts outwards when an asperity enters. This effect gives an additional reduction to the film thickness oscillations that are eventually propagated into the contact. Using the (extended) perturbation approach of Hooke [22] the effect of different alternative inlet conditions on the reduced waviness amplitude can be analyzed. This is the subject of ongoing research. However, preliminary results show that the effect depends on the exact condition: fixed position, fixed inlet film thickness or flow continuity condition, where the last situation seems to be the most realistic.

Meanwhile a crude way to scale the results may be to replace the parameter $\nabla$ by a parameter $\tilde{\nabla}$ that is corrected for the effect of starvation using the reduction of the entire film thickness level that is caused by the limited oil supply:

$$
\widetilde{\nabla}=\nabla\left(H_{c f} / H_{c}\right)^{\phi}
$$

From a practical point of view this is a convenient extension as for the the ratio $H_{c} / H_{c f}$ an approximate formula is available, see Eq. (11). Figure 8 shows the results obtained presented as a function of $\tilde{\nabla}$ taking $\phi=3 / 2$. It appears that for both transverse and isotropic waviness the results for the starved problem scale quite well on and near the results for the fully flooded case. The scaling is better for the severely starved cases than for the less starved cases. The observed spread is partly explained by the fact that the real mechanism is more complex than suggested by this simple scaling. In addition there will be some effect of numerical inaccuracy and of non-linearity. For small $H_{\text {oil }}$ the value $A_{i}=H_{c f} / 5$ is rather large compared to $H_{c}$ and when the waviness amplitude is of the same order of magnitude as the starved film thickness the deformed pattern is not exactly harmonic anymore. Finally, other values of $\phi$ may give a better result but the value of $3 / 2$ is preferred because this is the same power as with which $\bar{\alpha}$ appears in $\nabla$.

\section{Conclusion}

By means of numerical simulations the deformation of transverse and isotropic harmonic waviness in EHL circular contacts under pure rolling has been studied in relation to the lubricant supply to the contact. For the fully flooded contact it was shown in earlier work that the deformation of harmonic waviness under pure rolling is determined by a single non-dimensional parameter which can be referred to as a non-dimensional wavelength. Short wavelengths deform very little and the deformation increases with increasing wavelength. A simple engineering formula was derived that by applying it to each Fourier component of a roughness profile can be used to compute (an approximation to) the deformed roughness patterns. With separate studies for line and elliptic contacts, and for different orientation of the waviness pattern it was shown that waviness deformation in EHL contacts is determined by a single unifying mechanism.

One of the few parameters not yet investigated was the effect of the lubricant supply, or starvation which has been the subject of this paper. Starvation was modelled by introducing an additional variable representing the fraction to which the film is filled with oil, which allows the boundary between pressurized and cavitated/ starved regions to be free. The steady state smooth surface problem has been discussed as an introduction showing the decrease of the entire film thickness level with decreasing lubricant supply. Next the results for the time dependent problem with the waviness moving through the contact were presented. It has been shown that the relative deformed amplitude of the waviness depends on the same non-dimensional wavelength parameter as before, but that for a larger degree of starvation the relative deformed amplitude is smaller, or, a smaller lubricant supply leads to a larger reduction of the waviness amplitude inside the contact. The exact physical mechanism causing this behavior is subject of further research. However, meanwhile it has been shown that to a good approximation the relative deformed amplitude of the starved problem can be predicted by the formula for the fully flooded problem if the generalized wavelength parameter is corrected using the ratio of the central film thickness for the fully flooded steady state contact to the central film thickness for the starved steady state smooth contact. As for this ratio an accurate approximation formula is available, it appears that also for starved contacts using a Fourier component wise approach a crude estimate of the deformed surface micro-geometry (roughness) inside a contact can be obtained quite easily now. 


\section{Nomenclature}

$a=$ Hertzian contact length $a=\left(3 F R / 2 E^{\prime}\right)^{1 / 3}$

$A_{i}=$ dimensionless amplitude

$A_{d}=$ dimensionless amplitude film thickness oscillations

$\mathcal{A}=$ amplitude function

$c=$ constant

$E=$ modulus of elasticity

$E^{\prime}=$ reduced modulus of elasticity $2 / E^{\prime}=\left(1-\nu_{1}^{2}\right) / E_{1}+\left(1-\nu_{2}^{2}\right) / E_{2}$

$f=$ roughness orientation function

$F=$ external nominal load

$G=$ dimensionless parameter $G=\alpha E^{\prime}$

$h=$ film thickness

$H=$ dimensionless film thickness $H=h R_{x} / a^{2}$

$H_{c}=$ dimensionless central film thickness

$H_{b}=$ dimensionless film thickness at inlet

$H_{c f}=$ dimensionless central film thickness fully flooded conditions

$H_{\text {oil }}=$ dimensionless oil layer thickness

$\mathcal{I}=$ integral

$h_{T}=$ dimensionless time step

$h_{X}, h_{Y}=$ dimensionless mesh size in $X, Y$

$L=$ dimensionless material parameter $L=G(2 U)^{1 / 4}$

$M=$ dimensionless load parameter $M=W(2 U)^{-3 / 4}$

$n_{X}, n_{Y}=$ number of gridpoints in $X, Y$

$p=$ pressure

$p_{h}=$ maximum Hertzian pressure $p_{h}=(3 F) /\left(2 \pi a^{2}\right)$

$P=$ dimensionless pressure $P=p / p_{h}$

$r=$ parameter $r=H_{\text {oil }} /\left(\bar{\rho}\left(p_{h}\right) H_{c f}\right)$

$R=$ reduced radius of curvature, $1 / R=1 / R_{x}+1 / R_{y}$

$R_{x}=$ reduced radius of curvature in $x, 1 / R_{x}=1 / R_{x 1}$ $+1 / R_{x 2}$

$R_{y}=$ reduced radius of curvature in $y, R_{y}=R_{x}$

$R_{x 1}, R_{x 2}=$ radius of curvature surface 1,2 in $x$

$R_{y 1}, R_{y 2}=$ radius of curvature surface 1,2 in $y$

$\mathcal{R}=$ film thickness ratio, $H_{c} / H_{c f}$

$S=$ dimensionless speed parameter $S=u_{1} / \bar{u}$

$t=$ time

$T=$ dimensionless time $T=t \bar{u} / a$

$u=$ surface velocity

$u_{s}=$ sum velocity $u_{s}=\left(u_{1}+u_{2}\right)$

$\bar{u}=$ average velocity $\bar{u}=\left(u_{1}+u_{2}\right) / 2$

$U=$ dimensionless speed parameter, $2 U=\left(\eta_{0} u_{s}\right) /\left(E^{\prime} R_{x}\right)$

$\mathcal{W}=$ surface waviness function

$W=$ dimensionless load parameter, $W=F /\left(E^{\prime} R_{x}^{2}\right)$

$x, x^{\prime}=$ coordinate in direction of rolling

$X, X^{\prime}=$ dimensionless coordinate, $X=x / a, X^{\prime}=x^{\prime} / a$

$y, y^{\prime}=$ coordinate perpendicular to $x$

$Y, Y^{\prime}=$ dimensionless coordinate, $Y=y / a, Y^{\prime}=y^{\prime} / a$

$z=$ viscosity index (Roelands)

$\alpha=$ pressure viscosity coefficient

$\bar{\alpha}=$ dimensionless parameter $\bar{\alpha}=\alpha p_{h}$

$\gamma=$ parameter in equation

$\delta=$ mutual approach

$\Delta=$ dimensionless mutual approach $\Delta=\delta R_{x} / a^{2}$

$\epsilon=$ coefficient in Reynolds' equation $\epsilon=\left(\bar{\rho} H^{3}\right) /(\bar{\eta} \bar{\lambda})$

$\phi=$ exponent

$\nu=$ poisson ratio

$\lambda=$ wavelength

$\lambda_{x}=$ wavelength in $x$ direction

$\lambda_{y}=$ wavelength in $y$ direction

$\bar{\lambda}=$ dimensionless speed parameter $\bar{\lambda}=6\left(\eta_{0} u_{s} R_{x}^{2}\right) /\left(a^{3} p_{h}\right)$

$\eta=$ viscosity $\nabla=$ generalized wavelength $\nabla=c(\lambda / a) \bar{\alpha}^{3 / 2} L^{-2}$

$\tilde{\nabla}=$ corrected Generalized wavelength $\tilde{\nabla}=c\left(H_{c f} / H_{c}\right)^{\phi}(\lambda / a) \bar{\alpha}^{3 / 2} L^{-2}$

$\bar{\eta}=$ dimensionless viscosity $\bar{\eta}=\eta / \eta_{0}$

$\rho=$ density

$\bar{\rho}=$ dimensionless density $\bar{\rho}=\rho / \rho_{0}$

$\Sigma=$ slip parameter $\Sigma=\left(u_{1}-u_{2}\right) / \bar{u}$

$\theta=$ fractional film content

$\omega=$ variable

$\zeta=$ variable

\section{Subscripts}

$0=$ constant, e.g., at ambient pressure

$1,2=$ surface 1,2

$a, b=$ inlet side, outlet side

$s=$ start, at $T=0$

\section{Relations Between Parameters}

$$
\begin{aligned}
& \bar{\alpha}=L / \pi(3 M / 2)^{1 / 3} \\
& \bar{\lambda}=\left(128 \pi^{3} / 3 M^{4}\right)^{1 / 3} \\
& \Sigma=2(S-1)
\end{aligned}
$$

\section{References}

[1] Chiu, Y. P., 1974, “An Analysis and Prediction of Lubricant Film Starvation in Rolling Contact Systems," ASLE Trans., 17(1), pp. 22-35.

[2] Guangteng, G., Cann, P. M., and Spikes, H. A., 1992, "A Study of Parched Lubrication," Wear, 153, pp. 91-105.

[3] Chevalier, F., 1996, "Modélisation des Conditions d'Alimentation en Lubrifiant dans les Contacts Elastohydrodynamiques Ponctuels," Ph.D. thesis, INSA de Lyon, France.

[4] Cann, P. M., Lubrecht, A. A., and Venner, C. H., 2001, “Grease Lubrication of Rolling Element Bearings, A Model Future?," Proceedings International Tribology Conference Nagasaki 2000, in press.

[5] Wedeven, L. D., Evans, D., and Cameron, A., 1971, "Optical Analysis of Ball Bearing Starvation,” ASME J. Lubr. Technol., pp. 349-363.

[6] Pemberton, J., and Cameron, A., 1976, “A Mechanism of Fluid Replenishment in Elastohydrodynamic Contacts," Wear, 37, pp. 185-190.

[7] Wolverigde, P. E., Baglin, K. P., and Archard, J. F., 1971, "The Starved Lubrication of Cylinders in Line Contact," Proc. IMechE, 198(81), pp. 11591169.

[8] Ranger, A. P., Ettles, C. M. M., and Cameron, A., "The Solution of the Point Contact Elastohydrodynamic Problem,"' Proc. R. Soc. London, Ser. A, 346, pp. 227-244.

[9] Hamrock, B. J., and Dowson, D., 1977, "Isothermal Elastohydrodynamic Lubrication of Point Contacts, Part IV-Starvation Results," ASME J. Tribol., pp. $15-23$.

[10] Elrod, H. G., and Adams, M. L., 1974, "A Computer Program for Cavitation and Starvation Problems," Proceedings of the 1st Leeds-Lyon Symposium on Tribology, pp. 37-41.

[11] Elrod, H. G., 1981, “A Cavitation Algorithm,” ASME J. Lubr. Technol., 103, pp. 350-354.

[12] Chevalier, F., Lubrecht, A. A., Cann, P. M. E., Colin, F., and Dalmaz, G., 1994 "Starved Film Thickness: A Qualitative Explanation," Proceedings of the 21st Leeds-Lyon Symposium on Tribology, Elseviers Tribology Series, 30, pp. 249257.

[13] Chevalier, F., Lubrecht, A. A., Cann, P. M. E., Colin, F., and Dalmaz, G., 1995, "Starvation Phenomena in EHL Point Contacts: Influence of Inlet Flow Distribution," Proceedings of the 22nd Leeds-Lyon Symposium on Tribology, Elseviers Tribology Series, 31, pp. 213-223.

[14] Chevalier, F., Lubrecht, A. A., Cann, P. M. E., Colin, F., and Dalmaz, G., 1998, "Film Thickness in Starved EHL Point Contacts," ASME J. Tribol., 120, pp. $126-133$.

[15] Cann, P. M. E., Chevalier, F., and Lubrecht, A. A., 1997, "Track Depletion and Replenishment in a Grease Lubricated Point Contact: A Quantitative Analysis," Proceedings 23rd Leeds-Lyon Symposium on Tribology, Elseviers Tribology Series, 32, pp. 405-414.

[16] Wijnant, Y. H., 1998, "Contact Dynamics in the Field of Elastohydrodynamic Lubrication," Ph.D. Thesis, University of Twente, Enschede, The Netherlands, ISBN 90-36512239.

[17] Wijnant, Y. H., and Venner, C. H., "Contact Dynamics in Starved Elastohydrodynamic Lubrication," Proceedings of the 25th Leeds-Lyon Symposium on Tribology, Elsevier Tribology Series, 36, pp. 705-716.

[18] Dumont, M.-L., Lugt, P. M., and Tripp, J. H., 2002, "Surface Feature Effects in Starved Circular EHL Contacts," ASME J. Tribol., in press.

[19] Venner, C. H., Couhier, F., Lubrecht, A. A., and Greenwood J., 1997, “Amplitude Reduction of Waviness in Transient EHL Line Contacts," Proceedings 23rd Leeds-Lyon Symposium on Tribology, Elseviers Tribology Series, 32, pp. $103-112$.

[20] Venner, C. H., Lubrecht, A. A., 1999, “Amplitude Reduction of Anisotropic Harmonic Surface Patterns in EHL Circular Contacts under Pure Rolling," 
Proceedings of the 25th 1998 Leeds-Lyon Symposium on Tribology, Elsevier Tribology Series, 36, pp. 151-162.

[21] Venner, C. H., Kaneta, M., and Lubrecht, A. A., 2000, "Surface Roughness in Elastohydrodynamically Lubricated Contacts," Proceedings of the 26th LeedsLyon Symposium on Tribology, Elsevier Tribology Series, 38, pp. 25-36.

[22] Hooke, C. J., 1997, "Surface Roughness Modification in Elastohydrodynamic Line Contacts operating in the Elastic Piezoviscous Regime," Proc. Inst. Mech. Eng., Part J: J. Eng. Tribol., 212, pp. 149-146.

[23] Hooke, C., and Venner, C. H., 2000, "Surface Roughness attenuation in line and point contacts," Proc. Inst. Mech. Eng., Part J: J. Eng. Tribol., 214, pp. 439-444.

[24] Masen, M. A., Venner, C. H., Lugt, P. M., and Tripp, J. H., 2001, "Effects of Surface Micro-Geometry on the Lift-Off Speed of an EHL Contact," STLE Tribol. Trans., in press.

[25] Bayada, G., Chambat, M., and El Aloui, M., 1990, "Variational Formulations and Finite Element Algorithms for Cavitation Problems," ASME J. Tribol., 112, pp. 398-403.

[26] Roelands, C. J. A., 1966, "Correlational Aspects of the Viscosity-TemperaturePressure Relationship of Lubricating Oils" Ph.D thesis, Technical University Delft, Delft, The Netherlands, (V. R. B., Groningen, The Netherlands).

[27] Dowson, D., and Higginson, G. R., 1966, Elastohydrodynamic Lubrication, The Fundamentals of Roller and Gear Lubrication, Pergamon Press, Oxford, Great Britain.
[28] Jakobsson, B., and Floberg, L., 1957, "The Finite Journal Bearing, Considering Vaporization,” Trans. Chalmers Univ. Tech. Göteborg, p. 190.

[29] Olsson, K., 1965, "Cavitation in Dynamically Loaded Bearings," Trans. Chalmers Univ. Tech. Göteborg, 308.

[30] Brandt, A., and Lubrecht, A. A., 1990, "Multilevel Matrix Multiplication and Fast Solution of Integral Equations," J. Comput. Phys., 90, pp. 348-370.

[31] Venner, C. H., and Lubrecht, A. A., 2000, Multilevel Methods in Lubrication, Elsevier Tribology Series, 37, ISBN 0-444-50503-2.

[32] Venner, C. H., and Morales Espejel, G. E., 1999, "Amplitude Reduction of Small Amplitude Waviness in Transient Elastohydrodynamically Lubricated Line Contacts," Proc. Inst. Mech. Eng., Part J: J. Eng. Tribol., 213, pp. $487-$ 504.

[33] Damiens, B., Venner, C. H. Cann, P. M. E., and Lubrecht, A. A., 2001, "Starved Lubrication of Elliptical E.H.D. Contacts," ASME J. Tribol., /in press.

[34] Hamrock, B. J., and Dowson, D., 1977, "Isothermal Elastohydrodynamic Lubrication of Point Contacts, Part III, Fully Flooded Results," ASME J. Lubr. Technol., 99, pp. 264-276.

[35] Nijenbanning, G., Venner, C. H., and Moes, H., 1994, "Film Thickness in Elastohydrodynamically Lubricated Elliptic Contacts," Wear, 176, pp. 217 229.

[36] Hooke, C. J., 1977, “The Elastohydrodynamic Lubrication of Heavily Loaded Contacts," Proc. Inst. Mech. Eng., Part J: J. Eng. Tribol., 19(4), pp. 149-156. 